\title{
Genome size evolution in pufferfish: an insight from BAC clone-based Diodon holocanthus genome sequencing
}

\author{
Baocheng Guo 1,2, Ming Zou'1,2, Xiaoni Gan1,2,3 and Shunping He*1
}

\begin{abstract}
Background: Variations in genome size within and between species have been observed since the $1950 \mathrm{~s}$ in diverse taxonomic groups. Serving as model organisms, smooth pufferfish possess the smallest vertebrate genomes. Interestingly, spiny pufferfish from its sister family have genome twice as large as smooth pufferfish. Therefore, comparative genomic analysis between smooth pufferfish and spiny pufferfish is useful for our understanding of genome size evolution in pufferfish.

Results: Ten BAC clones of a spiny pufferfish Diodon holocanthus were randomly selected and shotgun sequenced. In total, $776 \mathrm{~kb}$ of non-redundant sequences without gap representing $0.1 \%$ of the $D$. holocanthus genome were identified, and 77 distinct genes were predicted. In the sequenced D. holocanthus genome, $364 \mathrm{~kb}$ is homologous with $265 \mathrm{~kb}$ of the Takifugu rubripes genome, and $223 \mathrm{~kb}$ is homologous with $148 \mathrm{~kb}$ of the Tetraodon nigroviridis genome. The repetitive DNA accounts for $8 \%$ of the sequenced D. holocanthus genome, which is higher than that in the T. rubripes genome (6.89\%) and that in the Te. nigroviridis genome (4.66\%). In the repetitive DNA, 76\% is retroelements which account for $6 \%$ of the sequenced $D$. holocanthus genome and belong to known families of transposable elements. More than half of retroelements were distributed within genes. In the non-homologous regions, repeat element proportion in D. holocanthus genome increased to 10.6\% compared with T. rubripes and increased to 9.19\% compared with Te. nigroviridis. A comparison of 10 well-defined orthologous genes showed that the average intron size (566 bp) in D. holocanthus genome is significantly longer than that in the smooth pufferfish genome (435 bp).

Conclusion: Compared with the smooth pufferfish, D. holocanthus has a low gene density and repeat elements rich genome. Genome size variation between $D$. holocanthus and the smooth pufferfish exhibits as length variation between homologous region and different accumulation of non-homologous sequences. The length difference of intron is consistent with the genome size variation between $D$. holocanthus and the smooth pufferfish. Different transposable element accumulation is responsible for genome size variation between $D$. holocanthus and the smooth pufferfish.
\end{abstract}

\section{Background}

Genome size, defined as the total amount of DNA contained within the haploid chromosome set of an organism, is not only one of the most fundamental genetic properties of living organisms, but also is one of the most mysterious biological traits. A well-known phenomenon is the "C-value paradox" [1] or "G-value/N-value paradox"

\footnotetext{
* Correspondence: heshunping@gmail.com

1 Fish Phylogenetics and Biogeography Group, Key Laboratory of Aquatic Biodiversity and Conservation, Institute of Hydrobiology, Chinese Academy of Sciences, Wuhan 430072, China

Full list of author information is available at the end of the article
}

$[2,3]$, which is the long-recognized lack of correlation between genome size and organism complexity [4]. A case in point is that some amoebas possess 200 times more DNA than humans [1]. Another striking characteristic of the genome size is that it can vary greatly among different taxonomic categories and even among closely related species. Genome size varies 250 -fold in arthropods, 350-fold in fish, 1,000-fold in angiosperms, 5,000fold in algae, and 5,800-fold in protozoans, and more than 200,000-fold in eukaryotes as reviewed by Gregory [5]. Genome size variation among closely related species is also prevalent and significant. For example, the fly genus 
Drosophila displays a twofold variation in genome size [6], whereas variation of genome size can achieve ninefold in a plant genus Crepsis [7].

Genome size variation appears to have resulted from a number of different processes over evolution time. In the short run, genome doubling or large-scale sequence duplication might be one of the most straightforward mechanisms underlying genome size variation $[1,8]$. The gain and loss of non-coding DNA are considered to be the main force behind the gradual accumulation of genome size variation over evolution time. For example, the correlation between genome size and transposable element amplification [9,10], and intron size variation [11-14], and gene duplication and pseudogenization [15] have been widely studied and verified across a broad phylogenetic range. Study has revealed that a massive loss of ancestral protein-coding genes has contributed to the reduction in the size of the chicken genome [16]. Overall, genome size variation is now recognized as reflecting the net effects of a collection of mechanisms that sometimes work antagonistically to expand and contract the genome, and is the result of that many forces affect collectively and operate heterogeneously among genomic regions $[17,18]$.

The comparative genomic analysis within closely related taxonomic groups is a powerful tool to uncover mechanisms of genome size variation, as studies in cotton [17-21] and Drosophila [6,22]. In this context, the pufferfish may be suitable organisms with which to study the genome size variation of vertebrates for the following reasons. The variation of genome size between tetraodontid and diodontid pufferfish presumably has resulted from a reduction of genome size in the smooth tetraodontid pufferfish relative to their spiny diodontid relatives since their divergence during the 50-70 million years ago (Figure 1) [23]. In the family Tetraodontidae, the smooth pufferfish have the smallest vertebrate genomes known to date, which are one-eighth the length of the human genome, with a haploid genome size 365 million bp in $T$. rubripes [13] and 340 million bp in Te. nigroviridis [24]. The spiny pufferfish in Diodontidae, the sister family of Tetraodontidae, have genomes that are roughly twice as large, about $800 \mathrm{Mb}[23,25,26]$. Mola mola (Molidae), a member of the closest outgroup to Tetraodontidae and Diodontidae, has a genome size around $800 \mathrm{Mb}[23,26]$. Therefore, the variation of genome size in pufferfish, especially the highly reduced genome size in the smooth pufferfish, makes pufferfish a good model system to study genome size shrinking in vertebrates. Furthermore, because of possessing small genomes, the smooth pufferfish (T. rubripes and Te. nigroviridis) have been used as model organisms and their genomes have been sequenced $[13,24]$. It is feasible to investigate the dynam-

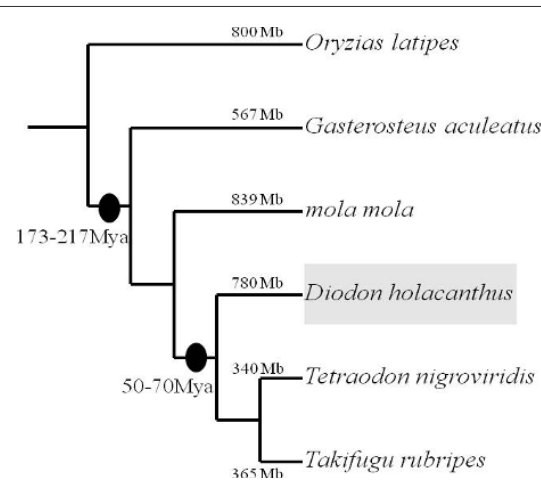

Figure 1 The evolutionary history of the pufferfish. The tree topology was modified according to Li et al. [52], and the divergent times were adopted from Steinke et al. [53] and Tyler and Santini [54]. The genome sizes of the species used in analysis are shown.

ics of genome size variation in large-scale genomic region rather than in particular genome region $[17,18]$.

By combining the results of the DNA renaturation kinetics analysis and rates of small $(<400 \mathrm{bp})$ insertions and deletions, Neafsey and Palumbi [27] proposed that the unequal rates of large insertions have contributed to the genome size variation between tetraodontid and diodontid pufferfish since their divergence, and genome compacting in the tetraodontid lineage is attributable to a reduction in the rate of large insertions rather than to an increase in large deletions. Aparicio et al [13] demonstrated that the genome of T. rubripes is compact partly because its introns are shorter than those of the human genome. To better understand the variation of genome size among pufferfish, it may be informative to compare closely related species within a phylogenetic framework using a comparative genomic approach by fully utilizing the whole genome sequence resources of $T$. rubripes and Te. nigroviridis.

Therefore, in this study, we have examined the genome of the spiny pufferfish $D$. holocanthus by sequencing bacterial artificial chromosomes (BAC) and conducted comparative genomic analyses with the genomes of the smooth pufferfish $T$. rubripes and Te. nigroviridis. The possible forces related to genome size variation were analyzed. Synteny analysis showed that less $50 \%$ of the sequenced $D$. holocanthus genomic sequences could be aligned with the genomes of tetraodontid pufferfish $T$. rubripes and Te. nigroviridis, which is consistent with their different genome size level. The repeat element component of the sequenced $D$. holocanthus genome was $7.94 \%$, which is higher than the total repeat element component of whole genomes of T. rubripes (6.89\%) and Te. nigroviridis (4.66\%). The differences of repeat element components in both the homologous regions and the non-homologous regions between $D$. holocanthus and 
tetraodontid pufferfish are large and significant, especially the proportion of retroelements. The differences of intron size between $D$. holocanthus and the smooth pufferfish are also significant. Our results showed that intron size variation was consistent with genome size variation between $D$. holocanthus and smooth pufferfish. We verified that different amount and content of transposable elements were responsible for the genome size variation between $D$. holocanthus and smooth pufferfish.

\section{Results}

\section{Sequence assembly and annotation}

A BAC library of the spiny pufferfish $D$. holocanthus was constructed and used for BAC clone selection in this study. Ten clones, containing sequences of around $100 \mathrm{~kb}$, were randomly selected and subsequently sequenced at the Beijing Genomics Institute. A total of 9,286 highquality reads produced a $2.84 \mathrm{Mb}$ data set. By combining with the estimated length of each BAC clone, we determined that the average coverage for draft sequences of these BAC clones is approximately 3.14-fold. Further assembly generated 49 scaffolds, ranging from $2.2 \mathrm{~kb}$ to $66.6 \mathrm{~kb}$ and representing a total of $776 \mathrm{~kb}$ of non-redundant sequences without gaps and $822 \mathrm{~kb}$ of non-redundant sequences with gaps of the $D$. holocanthus genome. The detailed sequencing information is given in Table 1.

The GC content of the sequenced D. holocanthus genome was $41.65 \%$. Gene annotation of these sequences predicted 87 putative genes, including alternatively splicing transcript forms. In the predicted gene set, 62 genes had complete open reading frames, ranging from $204 \mathrm{bp}$ to $13.8 \mathrm{~kb}$ with an average length of $1517 \mathrm{bp}$ and 8 coding exons per gene on average. In the predicted gene set, totally 619 complete exons existed and had an average length of $194 \mathrm{bp}$ and 532 introns had an average length of $845 \mathrm{bp}$. The coding regions accounted for approximately $120 \mathrm{~kb}$, or $15.5 \%$ of the sequenced $D$. holocanthus genome in total. In the predicted gene set, 27 of the predicted genes that were predicted by both FgenesH 2.6 [28] and GENSCAN 1.0 [29] had no significant hits when searched against the non-redundant GenBank protein database with BLASTP [30]. This subset of genes was named as novel predicted proteins. Names of remaining gene-encoded proteins were assigned according to the BLASTP searches. Possible alternatively spliced forms of 10 genes were annotated in the predicted gene set. Finally, a total of 77 distinct genes with either complete or incomplete coding sequences (CDS) were found in the sequenced BAC clones dataset. Detailed information regarding the predicted gene set of the spiny pufferfish is given in Table 2.

\section{Comparative mapping and synteny identification}

To identify homologous regions and sequence similarity patterns between the sequenced genome of $D$. holocanthus and the genomes of other species, we performed BLASTN searches against genomes of T. rubripes, Te. nigroviridis, Gasterosteus aculeatus, and Oryzias latipes. The genomic synteny regions between the sequenced genome of $D$. holocanthus and the genomes of other model fish were listed in Table 3 . The T. rubripes genome had the highest sequence similarity to the sequenced $D$. holocanthus genome, and $46.9 \%$ of the sequenced BAC sequences could be aligned with the genome of $T$. rubripes, $28.8 \%$ with the Te. nigroviridis genome, $22.1 \%$ with the G. aculeatus genome, and $20.9 \%$ with the O. latipes genome. Based on the results of the BLASTN searches (see Materials and Methods), 30 scaffolds of the

Table 1: Sequencing information of each BAC clone.

\begin{tabular}{|c|c|c|c|c|c|c|}
\hline$B A C I D$ & $\begin{array}{c}\text { Read } \\
\text { numbers }\end{array}$ & $\begin{array}{c}\text { Read } \\
\text { lengths(bp) }\end{array}$ & $\begin{array}{l}\text { Assembled } \\
\text { length(bp) }\end{array}$ & $\begin{array}{c}\text { Estimated } \\
\text { size }(k b)\end{array}$ & Coverage & N50 (bp) \\
\hline ctfa & 1020 & 311030 & 87525 & 90 & 3.46 & 78386 \\
\hline $\mathrm{ctfb}$ & 1013 & 335538 & 103323 & 110 & 3.05 & 67071 \\
\hline $\mathrm{ctfc}$ & 1000 & 324833 & 99742 & 95 & 3.42 & 51904 \\
\hline $\mathrm{ctfd}$ & 996 & 303386 & 89282 & 90 & 3.37 & 54060 \\
\hline ctfe & 890 & 279782 & 80143 & 100 & 2.80 & 41374 \\
\hline $\mathrm{ctff}$ & 881 & 251821 & 75743 & 80 & 3.15 & 40883 \\
\hline $\mathrm{ctfg}$ & 794 & 255225 & 62595 & 90 & 2.84 & 44454 \\
\hline ctfh & 942 & 281266 & 85394 & 80 & 3.52 & 66638 \\
\hline$c t f j$ & 655 & 186358 & 62614 & 70 & 2.66 & 39606 \\
\hline ctfk & 1095 & 315029 & 85969 & 100 & 3.15 & 43834 \\
\hline Total & 9286 & 2844268 & 832330 & & 3.14 & \\
\hline
\end{tabular}

Coverage, regarded as sequencing redundancy was calculated as the ratio of the total read length to the assembled length. N50 length is the size $x$ such that $50 \%$ of the assembly is in units of length at least $x$. 
Table 2: Summary of the predicted genes in each BAC clone.

\begin{tabular}{|c|c|c|c|}
\hline BACID & Scaffold ID & GenBank Acc. No. & Gene Name \\
\hline \multirow[t]{3}{*}{ ctfa } & Scaffold 000001 & $\underline{\text { GU002104 }}$ & $\begin{array}{l}\text { ring finger protein } 17 \text {; centromere protein } \mathrm{J} \text {; similar to transcription termination } \\
\text { factor, RNA polymerase II }\end{array}$ \\
\hline & Scaffold000002 & $\underline{\text { GU002105 }}$ & hypothetical protein LOC334519; unnamed protein product \\
\hline & Scaffold000003 & $\underline{\text { GU002106 }}$ & similar to ReO_6; similar to ring finger protein 17 \\
\hline \multirow[t]{5}{*}{$\mathrm{ctfb}$} & Scaffold000001 & $\underline{\text { GU002107 }}$ & $\begin{array}{l}\text { similar to GF20795; similar to KH domain-containing, RNA-binding, signal } \\
\text { transduction-associated protein 2; EGF-like domain-containing protein; novel } \\
\text { protein similar to vertebrate PHDfinger protein } 3 \text { (PHF3) }\end{array}$ \\
\hline & Scaffold000002 & $\underline{\text { GU002108 }}$ & \\
\hline & Scaffold000003 & $\underline{\text { GU002109 }}$ & $\begin{array}{l}\text { similar to KH domain-containing, RNA-binding, signal transduction-associated } \\
\text { protein } 2\end{array}$ \\
\hline & Scaffold000004 & $\underline{\text { GU002110 }}$ & similar to DNA primase large subunit(58 kDa) \\
\hline & Scaffold000005 & $\underline{\text { GU002111 }}$ & \\
\hline \multirow[t]{4}{*}{$\mathrm{ctfc}$} & Scaffold 000001 & $\underline{\text { GU002112 }}$ & $\begin{array}{l}\text { novel predicted protein; similar to neuron navigator } 1 \text {; similar to cysteine and } \\
\text { glycine-rich protein } 2\end{array}$ \\
\hline & Scaffold000002 & $\underline{\text { GU002113 }}$ & $\underline{\text { similar to cardiac troponin } \mathrm{T}}$; similar to plakophilin 1 \\
\hline & Scaffold000003 & $\underline{\text { GU002114 }}$ & similar to TEA domain family member 3 \\
\hline & Scaffold000004 & $\underline{\text { GU002115 }}$ & similar to troponin I, slow skeletal muscle \\
\hline \multirow[t]{5}{*}{ ctfd } & Scaffold 000001 & $\underline{\text { GU002116 }}$ & similar to neurocan; novel predicted protein \\
\hline & Scaffold000002 & $\underline{\text { GU002117 }}$ & $\begin{array}{l}\text { unnamed protein product; novel predicted protein; similar to hypothetical } \\
\text { LOC100002099; similar to deoxyhypusine hydroxylase/monooxygenase; } \\
\text { transmembrane } 6 \text { superfamily member 1-like }\end{array}$ \\
\hline & Scaffold000003 & $\underline{\text { GU002118 }}$ & similar to phosphodiesterase 4D-interacting protein; unnamed protein product \\
\hline & Scaffold000004 & $\underline{\text { GU002119 }}$ & \\
\hline & Scaffold000005 & $\underline{\text { GU002120 }}$ & unnamed protein product \\
\hline \multirow[t]{3}{*}{ ctfe } & Scaffold000001 & $\underline{\text { GU002121 }}$ & glutathione S-transferase theta; novel predicted protein \\
\hline & Scaffold000002 & $\underline{\text { GU002122 }}$ & $\begin{array}{l}\text { novel predicted protein; d-dopachrome decarboxylase; similar to coiled-coil- } \\
\text { helix-coiled-coil-helix domain containing 10; zinc finger 214-contain protein }\end{array}$ \\
\hline & Scaffold000003 & $\underline{\text { GU002123 }}$ & \\
\hline \multirow[t]{9}{*}{ ctff } & Scaffold 000001 & $\underline{\text { GU002124 }}$ & $\begin{array}{l}\text { similar to human adrenergic receptor kinase } 2 \text {; similar to human adrenergic } \\
\text { receptor kinase } 2\end{array}$ \\
\hline & Scaffold000002 & $\underline{\text { GU002125 }}$ & similar to beta-adrenergic kinase 2 ; novel predicted protein \\
\hline & Scaffold000003 & $\underline{\text { GU002126 }}$ & similar to myosin-like protein \\
\hline & Scaffold000004 & $\underline{\text { GU002127 }}$ & similar to beta-adrenergic receptor kinase 2 \\
\hline & Scaffold000005 & $\underline{\text { GU002128 }}$ & similar to beta-adrenergic receptor kinase 2 \\
\hline & Scaffold000006 & $\underline{\text { GU002129 }}$ & \\
\hline & Scaffold000007 & $\underline{\text { GU002130 }}$ & \\
\hline & Scaffold000008 & $\underline{\text { GU002131 }}$ & $\underline{\text { claudin } 5 \mathrm{~b}}$ \\
\hline & Scaffold000009 & $\underline{\text { GU002132 }}$ & novel predicted protein \\
\hline \multirow[t]{3}{*}{ ctfg } & Scaffold 000001 & $\underline{\text { GU002133 }}$ & novel predicted protein; similar to tetraspanin $18 ;$ novel predicted protein \\
\hline & Scaffold000002 & $\underline{\text { GU002134 }}$ & similar to erythrocyte membrane protein 3 ; novel predicted protein \\
\hline & Scaffold000003 & $\underline{\text { GU002135 }}$ & aristaless-like homeobox 4 \\
\hline \multirow[t]{3}{*}{ ctfh } & Scaffold000001 & GU002136 & $\begin{array}{l}\text { novel predicted protein; similar to myocyte enhancer factor } 2 \mathrm{a} \text {; novel predicted } \\
\text { protein; similar to multiple } \mathrm{C} 2 \text { and transmembrane domain-containing protein } 2\end{array}$ \\
\hline & Scaffold000002 & $\underline{\text { GU002137 }}$ & novel predicted protein; novel predicted protein; novel predicted protein \\
\hline & Scaffold000003 & $\underline{\text { GU002138 }}$ & \\
\hline ctfj & Scaffold 000001 & $\underline{\text { GU002139 }}$ & $\begin{array}{l}\text { novel predicted protein; novel predicted protein; similar to sterile alpha motif } \\
\text { domain containing 3; similar to zinc finger protein }\end{array}$ \\
\hline
\end{tabular}


Table 2: Summary of the predicted genes in each BAC clone. (Continued)

\begin{tabular}{llll}
\hline Scaffold000002 & $\underline{\text { GU002140 }}$ & $\begin{array}{l}\text { novel predicted protein; novel predicted protein } \\
\text { unnamed protein product } \\
\text { Scaffold000003 }\end{array}$ & $\underline{\text { GU002141 }}$ \\
Scaffold000004 & $\underline{\text { GU002142 }}$ & $\begin{array}{l}\text { Dexamethasone-induced Ras-related protein 1 precursor } \\
\text { Scaffold000005 }\end{array}$ & $\underline{\text { GU002143 }}$ \\
Scaffold000006 & $\underline{\text { GU002144 }}$ & \\
ctfk & Scaffold000001 & $\underline{\text { GU002145 }}$ & $\underline{\text { similar to calcium-transporting ATPase 2C1 isoform 1a; similar to aldehyde }}$ \\
& & $\underline{\text { dehydrogenase 5 family, member A1; similar to KIAA0319; Similar to DENN/ }}$ \\
Scaffold000002 & $\underline{\text { GU002146 }}$ & novel predicted protein; similar to calcium-transporting ATPase 2C1 \\
Scaffold000003 & $\underline{\text { GU002147 }}$ & novel predicted protein \\
Scaffold000004 & $\underline{\text { GU002148 }}$ & \\
Scaffold000005 & $\underline{\text { GU002149 }}$ & \\
Scaffold000006 & $\underline{\text { GU002150 }}$ & \\
Scaffold000007 & $\underline{\text { GU002151 }}$ & novel predicted protein \\
Scaffold000008 & $\underline{\text { GU002152 }}$ & \\
\hline
\end{tabular}

Novel predicted proteins represent predicted genes that had no significant hits when searched against the non-redundant GenBank protein database using BLASTP. Genes used for the comparisons of intron size variation are underlined.

sequenced BAC clones had syntenic regions and were localized on 12 scaffolds in T. rubripes, and the total length of the homologous regions was $364 \mathrm{~kb}$ in $D$. holocanthus and was $265 \mathrm{~kb}$ in T. rubripes; 25 BACsequenced scaffolds were localized on chromosome 2, 12, 13, and 17, and Un_random region in Te. nigroviridis, and the total length of the homologous regions was $223 \mathrm{~kb}$ in D. holocanthus and was $148 \mathrm{~kb}$ in Te. nigroviridis; 26 BAC-sequenced scaffolds were localized on 10 groups and 2 scaffolds in G. aculeatus, and the total length of the homologous regions was $171 \mathrm{~kb}$ in $D$. holocanthus and was $168 \mathrm{~kb}$ in G. aculeatus; 24 BAC-sequenced scaffolds were localized on chromosome 1, 5, 6, 12, 15, and 17, and scaffold 1954 and 4255 in O. latipes, and the total length of the homologous regions was $162 \mathrm{~kb}$ in $D$. holocanthus and was $159 \mathrm{~kb}$ in O. latipes. Figure 2 shows the synteny relationships of scaffolds of clone ctfh in the spiny pufferfish with T. rubripes and Te. nigroviridis. Fifteen BACsequenced scaffolds of the sequenced $D$. holocanthus genome were sequence-conserved and could be localized on all genomes of the other four model fish species. Eleven BAC-sequenced scaffolds, representing $100 \mathrm{~kb}$ or $12.1 \%$ of the sequenced $D$. holocanthus genome, had no homologous regions in the other four fish genomes. Fourteen BAC-sequenced scaffolds, representing a total length of $100 \mathrm{~kb}$ sequences or $12.2 \%$ of the sequenced $D$. holocanthus genome, could not be localized on the genomes of T. rubripes and Te. nigroviridis, but two of these sequences had homologous regions in G. aculeatus or O. latipes. Ten BAC-sequenced scaffolds displayed homologous relationships to the specific regions of the $T$. rubripes genome but had no synteny regions in the genome of Te. nigroviridis, whereas five BAC-sequenced scaffolds can be localized on the genome of Te. nigroviridis but had no synteny regions in the genome of $T$. rubripes.

\section{Intron size variation}

To investigate size intron variation between $D$. holocanthus and the smooth pufferfish, pairwise comparisons were performed. The predicted gene dataset of the sequenced $D$. holocanthus genome contained a total of 692 complete exons with an average length $208 \mathrm{bp}$ and 621 introns with an average length $868 \mathrm{bp}$. To accurately estimate size variations of intron of these species, the structures of the predicted genes with complete open reading frames were re-determined using GeneWise [31], a widely used tool that exquisitely predicted gene structure based on similarity searches against proteins. Finally, 10 genes (see Table 2) with high reliability (GeneWise score $>100$ ) were selected from the predicated gene dataset for intron size comparisons. After the introns with sequence gaps were excluded, a total of 125 gap-free introns were used for comparison with their orthologues in the other model fish species. Orthologous genes were retrieved directly from the Ensembl ortholog prediction dataset (release 52) in T. rubripes, Te. nigroviridis, and G. aculeatus.

The length distribution patterns of intron size are shown in Figure 3. The numbers and mean lengths of the intron size are listed in Table 4. The modal values of intron size appear in the range of 1-100 bp in all of these four species, with $83 \%$ of introns in T. rubripes and $76 \%$ of introns in Te. nigroviridis $<500 \mathrm{bp}$, whereas only $62 \%$ of 


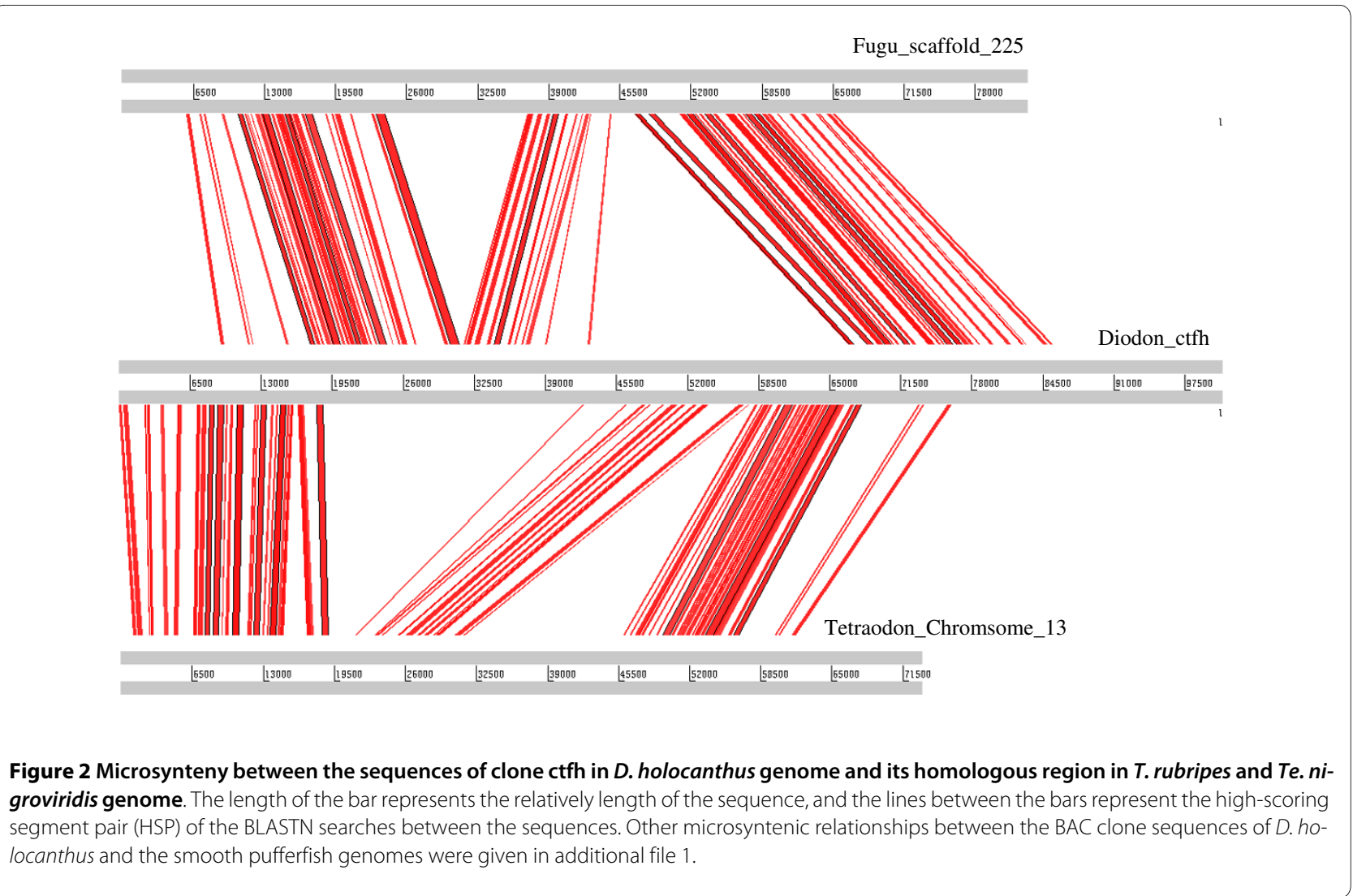

introns in D. holocanthus and 66\% of introns in G. aculeatus are shorter than $500 \mathrm{bp}$. The proportion of intron longer than 1000 bp was $7.1 \%$ in T. rubripes, $8.9 \%$ in Te. nigroviridis, $13.6 \%$ in D. holocanthus, and $18.9 \%$ in G. aculeatus. The mean length of intron in the T. rubripes genome was $435.2 \mathrm{bp}$ and was almost equal to that (434.9 $\mathrm{bp})$ in the Te. nigroviridis genome. The mean intron length in D. holocanthus is 566 bp which is obviously greater than that in the smooth pufferfish. The mean intron length in three-spined stickleback is clearly greater than that in the pufferfish. Because of the non-normal distribution of intron size (Kolmogorov-Smirnov normality test, $p=4.82 \mathrm{e}-007)$, the Wilcoxon signed rank test, a non-parametric test, was adopted and detected a significant differences in the intron lengths between $D$. holocanthus and T. rubripes ( $p=2.96 \mathrm{e}-022)$, between $D$. holocanthus and Te. nigroviridis $(P=5.30 \mathrm{e}-003)$, between $D$. holocanthus and G. aculeatus $(P=2.52 \mathrm{e}-013)$, between G. aculeatus and T. rubripes $(P=1.31 \mathrm{e}-019)$, and between G. aculeatus and Te. nigroviridis $(P=1.56 \mathrm{e}-006)$.

\section{Repetitive elements in pufferfish genome}

The different accumulation of repeat elements is recognized as a prominent force in genome size variation. Therefore, we examined the sequenced BAC clones and the smooth pufferfish genomes for evidence of repeat elements (Table 5). In total, 431 tracts of repeat element were detected in the sequenced spiny pufferfish genome. The total length of repeat elements was approximately 62 $\mathrm{kb}$ and accounted for $7.94 \%$ of the sequenced BAC clones. No small RNA or satellite repeats were detected. Simple repeats and low complexity repeats accounted for $0.75 \%$ and $0.46 \%$ of the sequenced spiny pufferfish genome, respectively. Our analysis result showed $6.73 \%$ of the sequenced BAC clones to match interspersed repeats. A large fraction of the interspersed repeats was contributed by retroelements $(6.00 \%$ of the BAC clone sequences), whereas $0.66 \%$ comprised DNA transposons and $0.07 \%$ unclassified interspersed repeats. We catalogued the transposable elements in the sequenced BAC clones in detail, and identified 21 subfamilies of transposable elements belonging to 12 known families (Table 6). The long interspersed repetitive elements (LINEs) constituted more than half the transposable elements and 3.44\% of the BAC sequences. Four LINE families, LINE1, LINE2, Rex1/Babar, and RTE, were detected, with 29 copies Expander representing the RTE family and 13 copies Maui belonging to the L2 family. Two short interspersed repetitive element (SINE) families V-SINE (81 copies) and Mermaid (25 copies), were found in the BAC clone sequences. One long terminal repeat (LTR) retrotransposons family, Gypsy/TY3 (4 copies), were identified. Four DNA transposon families, hAT-Charlie, Harbinger, Tc1- 
Table 3: Locations of each BAC on the genomes of different species

\begin{tabular}{|c|c|c|c|c|c|}
\hline BACID & Scaffold & T.rubripes & Te. nigroviridis & G. aculeatus & O. latipes \\
\hline \multirow[t]{3}{*}{$\mathrm{ctfa}$} & Scaffold000001 & scaffold_38 & Chromosome_2 & scaffold_115 & Chromosome_21 \\
\hline & Scaffold000002 & scaffold_38 & & groupXIV & \\
\hline & Scaffold000003 & & & & \\
\hline \multirow[t]{5}{*}{$\mathrm{ctfb}$} & Scaffold000001 & scaffold_24 & Chromosome_17 & groupVI & Chromosome_15 \\
\hline & Scaffold000002 & scaffold_24 & Chromosome_17 & groupXI & \\
\hline & Scaffold000003 & scaffold_24 & Chromosome_17 & groupVI & Chromosome_15 \\
\hline & Scaffold000004 & scaffold_24 & Chromosome_17 & groupVI & Chromosome_15 \\
\hline & Scaffold000005 & & & & scaffold3867 \\
\hline \multirow[t]{4}{*}{$\mathrm{ctfc}$} & Scaffold000001 & scaffold_635 & Un_random & groupXVII & Chromosome_5 \\
\hline & Scaffold000002 & scaffold_47 & Un_random & groupXVII & \\
\hline & Scaffold000003 & scaffold_47 & Un_random & groupXVII & Chromosome_5 \\
\hline & Scaffold000004 & & & groupXVII & Chromosome_5 \\
\hline \multirow[t]{5}{*}{$\mathrm{ctfd}$} & Scaffold000001 & scaffold_200 & & grouplll & Chromosome_17 \\
\hline & Scaffold000002 & scaffold_200 & & grouplll & Chromosome_17 \\
\hline & Scaffold000003 & scaffold_200 & & groupllI & Chromosome_17 \\
\hline & Scaffold000004 & scaffold_200 & Un_random & grouplll & Chromosome_17 \\
\hline & Scaffold000005 & scaffold_200 & & groupllI & \\
\hline \multirow[t]{3}{*}{ ctfe } & Scaffold000001 & & & & \\
\hline & Scaffold000002 & scaffold_589 & Un_random & & \\
\hline & Scaffold000003 & & & & \\
\hline \multirow[t]{9}{*}{$\mathrm{ctff}$} & Scaffold000001 & scaffold_27 & & & Chromosome_12 \\
\hline & Scaffold000002 & scaffold_27 & & & \\
\hline & Scaffold000003 & scaffold_27 & Un_random & & Chromosome_12 \\
\hline & Scaffold000004 & scaffold_27 & & & \\
\hline & Scaffold000005 & scaffold_27 & & & \\
\hline & Scaffold000006 & scaffold_27 & & & Chromosome_12 \\
\hline & Scaffold000007 & & & & \\
\hline & Scaffold000008 & scaffold_50 & Chromosome_12 & & \\
\hline & Scaffold000009 & & & & \\
\hline \multirow[t]{3}{*}{$\mathrm{ctfg}$} & Scaffold000001 & scaffold_2 & Chromosome_13 & groupXIX & Chromosome_6 \\
\hline & Scaffold000002 & & & & \\
\hline & Scaffold000003 & scaffold_2 & Chromosome_13 & groupXIX & Chromosome_6 \\
\hline \multirow[t]{3}{*}{$\mathrm{ctfh}$} & Scaffold000001 & scaffold_225 & Chromosome_13 & groupXIX & Chromosome_6 \\
\hline & Scaffold000002 & scaffold_225 & Chromosome_13 & groupXIX & Chromosome_6 \\
\hline & Scaffold000003 & scaffold_225 & Chromosome_13 & groupXIX & Chromosome_6 \\
\hline \multirow[t]{6}{*}{$\mathrm{ctfj}$} & Scaffold000001 & & Un_random & groupXIII & Chromosome_1 \\
\hline & Scaffold000002 & & Chromosome_2 & groupXIII & \\
\hline & Scaffold000003 & & Un_random & & \\
\hline & Scaffold000004 & scaffold_3 & Chromosome_2 & groupV & scaffold4255 \\
\hline & Scaffold000005 & & Chromosome_2 & & \\
\hline & Scaffold000006 & & & & \\
\hline \multirow[t]{8}{*}{$\mathrm{ctfk}$} & Scaffold000001 & scaffold_270 & Un_random & groupXX & scaffold1954 \\
\hline & Scaffold000002 & scaffold_270 & Un_random & groupXX & scaffold1954 \\
\hline & Scaffold000003 & & Chromosome_2 & & \\
\hline & Scaffold000004 & & & & \\
\hline & Scaffold000005 & & & & \\
\hline & Scaffold000006 & & & & \\
\hline & Scaffold000007 & & & & \\
\hline & Scaffold000008 & & & scaffold_56 & \\
\hline
\end{tabular}




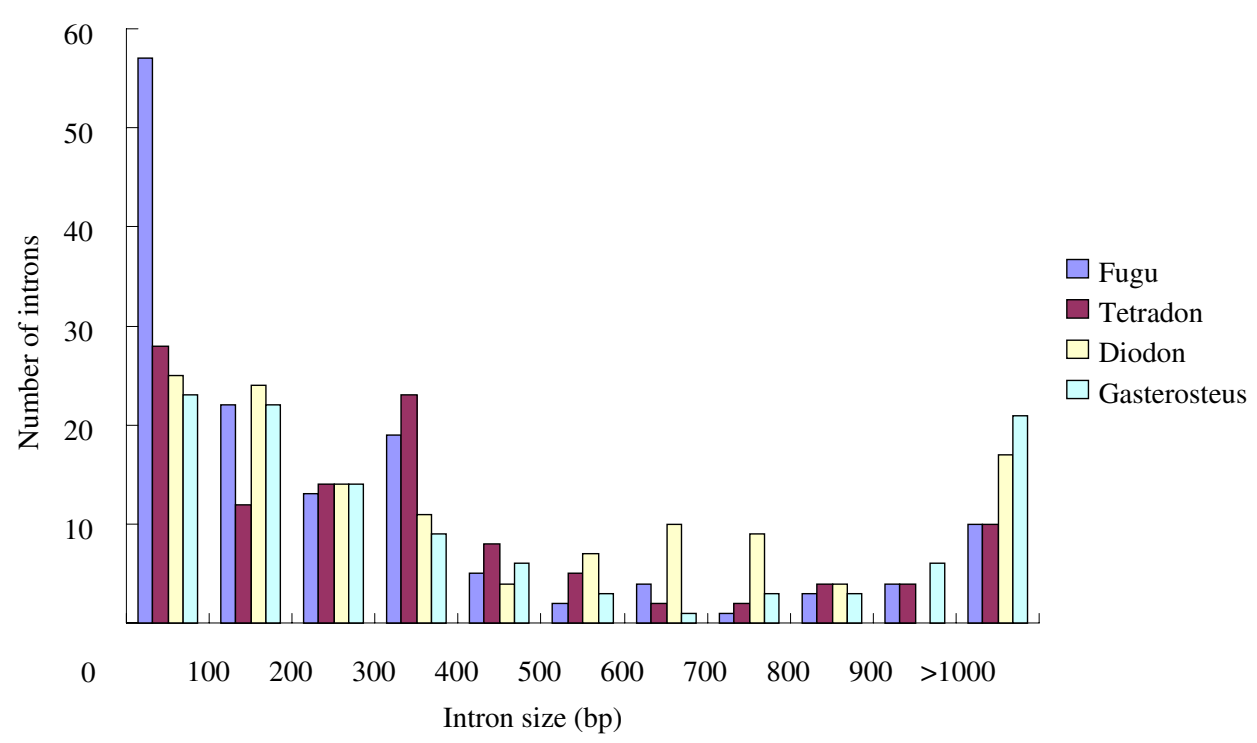

Figure 3 Distribution patterns of intron size. Distributions of the intron lengths used for the comparison in D. holocanthus (Diodon), T. rubripes (Fugu), Te. nigroviridis (Tetraodon), and G. aculeatus (Gasterosteus).

mariner, and Tc2, were detected and accounted for $0.66 \%$ of the BAC clone sequences. Our analysis showed that most of the repeat elements were distributed within genes (e.g., 67 of 106 copies of SINEs fall into introns, as do 77 of 135 copies of simple repeats) in the sequenced region of the $D$. holocanthus genome.

To accurately estimate the contribution of repeat elements to pufferfish genome size variation, we compared the proportion of repeat elements in the homologous regions between the spiny pufferfish $D$. holocanthus and the smooth pufferfish based on the synteny analysis (Table 5). In the homologous regions between D. holocanthus and T. rubripes, repeat elements accounted for approximately $19 \mathrm{~kb}$ and $5.16 \%$ of the sequences in $D$. holocanthus, whereas the total length of the repeat elements was $6,457 \mathrm{bp}$, accounting for $2.43 \%$ of the homologous regions in T. rubripes. In the homologous regions between $D$. holocanthus and Te. nigroviridis, repeat elements accounted for approximately $11 \mathrm{~kb}$ and $4.87 \%$ of the sequences in $D$. holocanthus, whereas accounted 4,304 bp or $2.93 \%$ of homologous sequences in Te. nigroviridis. No small RNA or satellite repeats were detected in their homologous regions. Although their sequence lengths were almost equal, the proportion of low complexity repeats in $D$. holocanthus was lower than those in the smooth pufferfish. The proportions of simple repeats in the smooth pufferfish were much higher than that in $D$. holocanthus. Unlike D. holocanthus, simple repeat elements contributed the major fraction (almost $80 \%$ ) of the repeat elements in homologous region of Te. nigroviridis. The proportion of simple repeats was $2.34 \%$ in the $T e$. nigroviridis, and was more than three times larger than it $(0.75 \%)$ in $D$. holocanthus. The total length of simple repeats was 3,435 bp in Te. nigroviridis and was 1,666 bp in D. holocanthus.

In the homologous regions between $D$. holocanthus and T. rubripes, the total length of interspersed repeats was approximately $15 \mathrm{~kb}$ and accounted for $4.05 \%$ of the homologous sequences in $D$. holocanthus, whereas in $T$. rubripes the length was 2,748 bp and accounted for $1.03 \%$ of the homologous regions. SINEs were absent from $T$. rubripes, but 39 copies of SINEs, including 30 copies of V-SINE and 9 copies of SINE/Mermaid, were identified in D. holocanthus. The total length of SINEs in D. holocanthus was $5,944 \mathrm{bp}$, accounting for $1.63 \%$ of the homologous regions. The proportion of the LINEs in $D$.

Table 4: Comparison of the average intron size and exon lengths of $D$. holocanthus, $T$. rubripes, and Te. nigroviridis.

\begin{tabular}{lllll}
\hline & D. holocanthus & T. rubripes & Te. nigroviridis & G.aculeatus \\
\hline Intron number & 125 & 140 & 111 & 110 \\
Average intron length (bp) & 566 & 435 & 435 & 722 \\
\hline
\end{tabular}


Table 5: Comparison of repetitive DNA sequence contents (\%) of D. holocanthus, T. rubripes, and Te. nigroviridis

\begin{tabular}{|c|c|c|c|c|c|c|c|}
\hline \multirow[t]{2}{*}{ Component } & \multicolumn{3}{|c|}{ D. holocanthus } & \multicolumn{2}{|c|}{ T. rubripes } & \multicolumn{2}{|c|}{ Te. nigroviridis } \\
\hline & WG & HR-fugu & HR-tetra & WG & HR-Dh & WG & HR-Dh \\
\hline Bases masked (\%) & 7.94 & 5.16 & 4.87 & 6.89 & 2.43 & 4.66 & 2.93 \\
\hline Interspersed repeats & 6.73 & 4.05 & 3.84 & 4.69 & 1.03 & 2.03 & 0.18 \\
\hline Retroelements & 6.00 & 3.76 & 3.36 & 3.39 & 0.33 & 1.37 & 0.08 \\
\hline SINES & 2.00 & 1.63 & 1.15 & 0.20 & 0.00 & 0.13 & 0.00 \\
\hline LINEs & 3.44 & 2.13 & 2.12 & 2.51 & 0.22 & 1.06 & 0.08 \\
\hline LTR elements & 0.55 & 0.00 & 0.00 & 0.68 & 0.11 & 0.19 & 0.00 \\
\hline DNA transposons & 0.66 & 0.29 & 0.48 & 1.05 & 0.61 & 0.45 & 0.00 \\
\hline Rolling-circles & 0.00 & 0.00 & 0.00 & 0.00 & 0.00 & 0.00 & 0.00 \\
\hline Unclassified & 0.07 & 0.00 & 0.00 & 0.25 & 0.09 & 0.21 & 0.10 \\
\hline Small RNA & 0.00 & 0.00 & 0.00 & 0.00 & 0.00 & 0.00 & 0.00 \\
\hline Satellites & 0.00 & 0.00 & 0.00 & 0.01 & 0.00 & 0.00 & 0.00 \\
\hline Simple repeats & 0.75 & 0.68 & 0.75 & 1.75 & 0.93 & 1.96 & 2.34 \\
\hline Low complexity & 0.46 & 0.43 & 0.28 & 0.44 & 0.47 & 0.67 & 0.41 \\
\hline
\end{tabular}

WG, sequenced whole genome dataset; HR-fugu, homologous region in the $D$. holocanthus genome compared with the T. rubripes genome; HR-tetra, homologous region in the $D$. holocanthus genome compared with the Te. nigroviridis genome; HR-Dh, homologous regions in the smooth pufferfish genomes compared with the $D$. holocanthus genome.

holocanthusis $2.13 \%$, which is almost 10 times higher than it $(0.22 \%)$ in $T$. rubripes. In D. holocanthus 21 partial copies of LINEs were detected, whereas there were only 3 partial copies in T. rubripes. No LTR retrotransposon was detected in $D$. holocanthus, whereas 2 copies were identified in T. rubripes. Unlike retroelements, the proportion of DNA transposons in T. rubripes was more than twice as high as that in D. holocanthus. The proportions of the interspersed repeats varied significantly in the homologous regions between $D$. holocanthus and Te. nigroviridis. In D. holocanthus, the proportion was $3.84 \%$ ( $8573 \mathrm{bp}$ ), with $1.15 \%$ of SINEs, $2.12 \%$ of LINEs, and $0.48 \%$ DNA transposons. In Te. nigroviridis, the proportion is only $0.18 \%$, with $0.08 \%$ of LINEs and $0.10 \%$ of unclassified interspersed repeat, whereas SINEs are absent. Similar to the distribution pattern in the sequenced $D$. holocanthus genome, most of the repeat elements in $D$. holocanthus fell within genes in the homologous regions shared with the smooth pufferfish, e.g., 27 out of 37 SINE copies were integrated within introns in its homologous regions shared with T. rubripes and 8 out of 17 copies occurred in the homologous region it shares with Te. nigroviridis.

\section{Discussion}

\section{Character of the $D$. holocanthus genome}

In recent years, the use of comparative genomic approach to study genome size variation within a phylogenetic framework has shed much light on the genome size varia- tion in closely related species, such as studies in cotton $[17,18,20,21,32]$, in rice [10], and in Drosophila [6,22]. Here, to study the genome size variation in pufferfish, especially the genome shrinking of the smooth pufferfish, a total length of $776 \mathrm{~kb}$ of non-redundant sequences or $0.1 \%$ of the spiny pufferfish $D$. holocanthus genome (780 $\mathrm{Mb}$ ) was sequenced and compared with the smooth pufferfish genomes. The GC level of the sequenced D. holocanthus genome $(41.65 \%)$ is within the vertebrate range, between $40 \%$ for Bos taurus and 48\% for Sus scrofa [33], but it is lower than it in the genome of $T$. rubripes (45.46\%) and Te. nigroviridis (46.43\%), whereas it is close to it in O. latipes genome $(40.46 \%)$. Because GC content represents gene density to some extent, the spiny pufferfish should have a lower gene density genome compared with the smooth pufferfish with compact genomes. This pattern is also supported by the proportion of coding region in the sequenced $D$. holocanthus genome. The coding region accounted for $15.5 \%$ of the assembled spiny pufferfish genome, whereas in Te. nigroviridis it accounted for $40 \%$ of the genome [24]. Our gene annotation results showed that the mean number of coding exons per gene with complete CDS on average (8 when untranslated regions are excluded) in the D. holocanthus genome is close to that in human genome (8.7) and mouse genome (8.4) [34], but is larger than that in the Te. nigroviridis (6.9) genome [24]. Assuming that fish and mammal genes have similar structures, this suggests that 
Table 6: Transposable elements in D. holocanthus and their classification

\begin{tabular}{|c|c|c|c|}
\hline Repeat classification & Distribution & Fugu members & Copy number \\
\hline \multicolumn{4}{|l|}{ SINEs } \\
\hline \multirow[t]{2}{*}{ V } & Vertebrates & FR2 & $63(46)$ \\
\hline & & TE & $18(11)$ \\
\hline \multirow[t]{2}{*}{ Mermaid } & Vertebrates & FR1c & $16(5)$ \\
\hline & & FR1d & $9(3)$ \\
\hline \multicolumn{4}{|c|}{ Non-LTR Retrotransposons } \\
\hline L1 & Vertebrates, plants & KibiFr1 & 2 \\
\hline \multirow[t]{2}{*}{ RTE } & Nematodes & Expander & 29 \\
\hline & & Expander2 & 2 \\
\hline L2 & Metazoa & Maui & 13 \\
\hline Rex1/Babar & Fish & Rex1_FurC & 7 \\
\hline \multicolumn{4}{|l|}{ LTR Retrotransposons } \\
\hline \multirow[t]{4}{*}{ Gypsy/TY3 } & Eukaryotes & Ronin1_I & 4 \\
\hline & & Ronin2_I & 1 \\
\hline & & Ronin3_I & 1 \\
\hline & & Samurai_I & 1 \\
\hline Penelope-like & Insects, fish & Bridge2(Xena) & 17 \\
\hline \multicolumn{4}{|l|}{ DNA transposons } \\
\hline hAT-Charlie & Mammals & Chaplin4_FR & $2(2)$ \\
\hline Harbinger & Nematodes, plants & Senkusha1A & $2(1)$ \\
\hline \multirow[t]{3}{*}{ Tc1-mariner } & Metazoa, plants & TC1_FR1 & $8(3)$ \\
\hline & & TC1_FR3 & $2(0)$ \\
\hline & & TC1_FR4 & $5(3)$ \\
\hline \multirow[t]{2}{*}{ Tc2 } & Nematodes & TC2_FR2a & $2(2)$ \\
\hline & & TC2_FR4 & $1(1)$ \\
\hline Unclassified & & & $6(1)$ \\
\hline
\end{tabular}

Numbers in parentheses represent the number of transposable elements distributed within gene.

our gene prediction results are trustworthy and that some annotated genes of Te. nigroviridis are partial or fragmental as suggested by Jaillon et al. (2004) [24]. Our repeat element analysis showed that the sequenced $D$. holocanthus genome contains more repeat elements, especially the interspersed elements, than do the smooth pufferfish genomes, which is consistent with the DNA renaturation analysis in previous study [27]. Thus, D. holocanthus has a lower gene density and repeat elements rich genome compared with smooth pufferfish. It is noteworthy that no inconsistency for the arrangement of scaffolds was detected during comparison with the smooth pufferfish genomes, which suggests that the order of assembled scaffolds is reliable and implies that negligible genomic rearrangement has occurred in the sequenced region of the $D$. holocanthus genome at the scale of the BAC clones $(\sim 100 \mathrm{~kb})$.

\section{Genome size evolution in pufferfish}

Variations in genome size within and between species have been observed since the $1950 \mathrm{~s}$ in diverse taxonomic groups. Interestingly, the smooth pufferfish have the smallest vertebrate genomes known to date, and evolved toward a high level of compact organization. Within the pufferfish, the genome of $D$. holocanthus is almost twofold larger than that of the smooth pufferfish. However, the difference of genome size between this spiny puffer and the smooth puffer does not appear to reflect a difference in ploidy [23], although the karyotype of D. holocanthus is unknown. The chromosome number (2n) ranges from 34 to 44 in the smooth pufferfish, and one spiny pufferfish $D$. bleekeri karyotyped to date has 46 chromosomes [35]. Parsimony analysis of the phylogenetic patterns of genome size in pufferfish suggests that the smooth pufferfish and the spiny pufferfish should have a plesiomorphic genome size of $800-900 \mathrm{Mb}$ and that the 
tiny genome of the smooth pufferfish is a derived character and unique to the smooth pufferfish [23]. Our synteny analysis supports this conclusion to some extent, insofar as doubled regions homologous of the smooth pufferfish genome were not detected in the sequenced $D$. holocanthus genome and less than half the BAC clone sequences can be aligned with the smooth pufferfish genomes. Thus, the almost twofold size difference between $D$. holocanthus and the smooth pufferfish genomes must be the result of other processes, which have contributed to this genome size variation over the long evolution timescales.

Previous studies have revealed that intron size variation is positively correlated with genome size variation $[11,12,22]$. Compared with human, the compact genome of $T$. rubripes is suggested to correlate positively with intron size shrinking [13,36]. In our analysis, we observed a correlation between the size of genome and intron length in pufferfish, with the smooth pufferfish having both smaller genomes and shorter introns compared with D. holocanthus having relative larger genome size and long introns. Statistical analysis showed that the difference of the average intron length between $D$. holocanthus (mean of $566 \mathrm{bp}$ ) and the smooth pufferfish (mean of 435 bp) was statistically significant. Although only a tiny fraction of the introns was sampled in our analysis, the nearly identical length distribution patterns (Figure 3) compared with that in the $T$. rubripes genome [13] suggested that our sampled data were not biased and the result was robust. Additionally, G. aculeatus was used as outgroup for intron size comparisons. Our results showed that the intron size in the G. aculeatus genome was significantly larger than that in pufferfish genomes, which suggested that different the intron size variation between the smooth pufferfish and the spiny pufferfish contributed to their genome size variation since their divergence with $G$. aculeatus. According to our analysis result, the intron length variation involved $31 \mathrm{Mb}$ sequence and accounts for approximately $7.21 \%$ of the genome size variation between $D$. holocanthus and smooth pufferfish, if 30,000 genes exist in pufferfish genome as suggested by Jaillon et al [24]. The correlation between the genome size and intron size in pufferfish is consistent with that observed in Drosophila [22], bird [11], and Muntiacus muntjak vaginalis [14], but differs from that in plants (e.g., Gossypium [21]). It seems that the correlation between intron and genome sizes depends on both the phylogenetic distance of the organisms studied and the time of their divergence. In fact, one explanation of to this phenomenon is that the rate of accumulation of insertions and deletions (indels) in intron sequence has varied in different organisms over evolution time $[37,38]$. For example, as one of the main insertion forces, transposable elements affect plant and mammalian genomes in different ways. Most transposable element insertions occur in the inter- genic regions of plant genomes $[39,40]$, but within intragenic regions (introns) of mammalian genomes [41]. As in the mammalian genomes, we found that most of the repeat elements in the sequenced $D$. holocanthus genome are mainly distributed in introns and have partially contributed to the difference of intron size between $D$. holocanthus and the smooth pufferfish. Another potential mechanisms responsible for the intron size variation between $D$. holocanthus and the smooth pufferfish may be the accumulation of small indels over their evolution time, as has occurred in the in cotton genus [32]. However, small indels cannot be detected in intron sequence between $D$. holocanthus and smooth pufferfish because of their relatively long divergence time (Figure 1). Thus, our comparison of the intron sizes of orthologous genes between $D$. holocanthus and the smooth pufferfish strongly supports the proposition that intron size variation resulting from different repeat element insertions and different accumulation of indels have contributed to genome size difference between $D$. holocanthus and the smooth pufferfish, which is consistent with the conclusion drawn in previous studies [11-14,22,36]. Overall, our results demonstrated that genes in the $D$. holocanthus genome are characterized by significant expansion in their intron size compared with the smooth pufferfish and accounted for genome size variation in the pufferfish. Genome size variation takes place also within genes in pufferfish.

In addition to intron size variation, the most potential force responsible to genome size variation is the differential accumulation and deletion of transposable elements, which have been observed and verified across a broad phylogenetic range of organisms including plants [20] and mammals [42,43]. A previous DNA renaturation analysis showed that middle-repetitive DNA was underrepresented in the smooth pufferfish genomes compared with a spiny pufferfish $(D$. hystrix) genome, and that a significantly greater abundance of a transposon-like repetitive DNA class existed in the spiny pufferfish genome relative to the smooth pufferfish genomes [27]. In our analysis, the proportion of repeat elements in the sequenced $D$. holocanthus genome (7.94\%) is higher than that in the smooth pufferfish genomes, with $6.89 \%$ in the T. rubripes genome and $4.66 \%$ in the Te. nigroviridis genome. Among the repeat elements, the fraction of interspersed repeats in the D. holocanthus genome was much higher than that in the smooth pufferfish genomes, but the proportion of simple repeats is lower. Our result is consistent with the result of DNA renaturation analysis [27]. We estimated here that the number of the interspersed repeats in the $D$. holocanthus genome $(193,000$, calculated from 193 copies in the $0.1 \%$ sequenced genome region) far exceeds that in the $T$. rubripes genome (46,000 copies) and that in the Te. nigroviridis 
genome $(19,000$ copies). To ensure that this pattern did not result from sample bias because only part of the $D$. holocanthus genome was available for analysis, we identified the repeat elements in the homologous regions between $D$. holocanthus and the smooth pufferfish genomes. This pattern recurred with an increasing discrepancy in the interspersed repeat fraction. In the homologous regions, the proportion of interspersed repeats in the $D$. holocanthus genome was nearly four times higher than that in the $T$. rubripes genome and more than 20 times higher than that in the Te. nigroviridis genome (Table 5). We estimated that the different profiles of repeat element sequences accounted for $12.5 \%$ of the homologous region variation between $D$. holocanthus and T. rubripes and for $8.59 \%$ between D. holocanthus and Te. nigroviridis. In the non-homologous regions, repeat element proportion in $D$. holocanthus genome increased to $10.6 \%$ compared with $T$. rubripes and increased to $9.19 \%$ compared with Te. nigroviridis. These are obviously higher than the proportion in the sequenced $D$. holocanthus genome (7.94\%), and are nearly twice as high as these in the homologous regions (5.16\% compared with T. rubripes and $4.87 \%$ compared with Te. nigroviridis). The increasing proportion of repeat elements in the non-homologous region indicates that the rates of repeat element accumulation were inconsistent across different $D$. holocanthus genomic regions. Distribution analysis result showed that transposable elements (e.g., SINEs) have accumulated both in the intergenic regions and introns, which have contributed to genome size variation between $D$. holocanthus and the smooth pufferfish. Previous studies have showed that the profiles of interspersed repeats differed in the smooth pufferfish genomes $[13,24]$. For example, the most common repeat in the T. rubripes genome was LINE-like element Maui, whereas in the Te. nigroviridis genome was DNA transposon-Buffy. Unlike in the smooth pufferfish genomes, the most common repeat in the sequenced $D$. holocanthus genome was V-SINE, a kind of non-autonomous retrotransposon. Imai et al. [44] also found that various repetitive elements accounted for genome size variation between $T$. rubripes and $O$. latipes by analyzing chromosome LG22 of O. latipes and its corresponding region in T. rubripes, which is consistent with our result. However, both the extent and types of repetitive elements which accounted for genome size variation between $T$. rubripes and $O$. latipes are different from our results. More than a half $(54 \%)$ of genome size variation between T. rubripes and $O$. latipes was contributed by various repetitive elements, which is much higher than that between pufferfish in our analysis. Instead of interspersed repeats differently accumulating, genome size variation mainly resulted from different accumulation of unclassified low copy repeats between $T$. rubripes and O. latipes.
In fact, the inconsistence between our results and Imai et al. [44] might be due to the following reasons. Firstly, different genomic regions were selected for studying. Our synteny analyses showed that selected genomic regions were not overlapped between our study and Imai et al. [44] (see Table 3). Secondly, the most conceivable reason is that results of genome size variation study rely on phylogenetic relationship and divergent time between selected species for comparison. T. rubripes diverged from O. latipes before 184 million years ago in Imai et al. [44], which is much earlier than the divergence time between $D$. holocanthus and T. rubripes (50-70 Mya) in our study (Figure 1). Although inconsistency exists, both our study and Imai et al. [44] supported that different content of repetitive elements accounted for genome size shrinking of smooth pufferfish. Thus, our analysis confirmed the previous DNA renaturation analysis [27] and demonstrated that different content of transposable elements have contributed to the genome size variation in the pufferfish.

Our synteny alignments of the sequenced BAC clones and the genomes of other fish species provided an overview of the genome size variation in the pufferfish. According to the synteny identification, the sequences of the $D$. holocanthus genome could be classified into the following two regions compared with smooth pufferfish genomes: the homologous region, which could be located onto smooth pufferfish genomes; and the non-homologous region, which had no homology with the smooth pufferfish genomes. We inferred that the length of the homologous region in $D$. holocanthus genome was at least 1.37 times longer than that in the $T$. rubripes genome (364 kb vs $265 \mathrm{~kb}$, respectively), and was 1.52 times longer than that in the Te. nigroviridis genome (223 kb vs $148 \mathrm{~kb}$, respectively). Therefore, we deduced that the length of the homologous region between $D$. holocanthus and T. rubripes was $364 \mathrm{Mb}$ in the D. holocanthus genome and was $266 \mathrm{Mb}$ in the T. rubripes genome, and that between $D$. holocanthus and Te. nigroviridis was 224 $\mathrm{Mb}$ in D. holocanthus and was $147 \mathrm{Mb}$ in Te. nigroviridis. This analysis implied that genome size variation between D. holocanthus and smooth pufferfish was partially exhibited as length variations between the homologous regions. Also the lengths of their non-homologous sequences are different; in particular the length of the non-homologous sequence in $D$. holocanthus is longer than that in smooth pufferfish. This is partially explained by the finding that repeat element proportion in D. holocanthus genome increased to $10.6 \%$ compared with $T$. rubripes and increased to $9.19 \%$ compared with Te. nigroviridis in the non-homologous regions. Interestingly, $T$. rubripes and Te. nigroviridis have such different amounts of sequence both in homologous and non- homologous region compared with $D$. holocanthus. With 2 smooth 
pufferfish sharing most of the biological traits and equal divergence time with $D$. holocanthus, one explanation for this result is different generation time between $T$. rubripes and Te. nigroviridis. The generation time of Te. nigroviridis is much shorter (about 1 year) compared with T. rubripes (3-4 years), which make they have different evolutionary rate and result in their different genome similarity compared with $D$. holocanthus. A fraction of $D$. holocanthus genomic sequences was not homologous with other fish genomes (e.g., G. aculeatus and O. latipes) and might be the $D$. holocanthus genome-specific sequence. This part of the $D$. holocanthus genome-specific sequence accounted for $12.1 \%$ or approximately 94 $\mathrm{Mb}$ of the $D$. holocanthus genome and contributed approximately $21.86 \%$ of genome size variation between D. holocanthus and the smooth pufferfish. Using G. aculeatus and O. latipes as reference species, we found that a fraction of the homologous region of the D. holocanthus genome $(0.1 \%)$ might have been lost in the two smooth pufferfish genomes. These results suggested that genome size variation between $D$. holocanthus and the smooth pufferfish was exhibited as the length variation in the homologous region and different levels of the nonhomologous sequence accumulation.

The interest in the genome size variation among the pufferfish mainly arises from that the smooth pufferfish have the smallest vertebrate genomes yet measured. If $D$. holocanthus and smooth pufferfish genomes are presently at equilibrium with regard to their size and the tiny genome is a derived character and unique to the smooth pufferfish [23], the difference in genome size between the pufferfish implies that an ancestral equilibrium was disturbed in the smooth tetraodontid lineage following its divergence from the spiny diodontids. The mechanisms of genome size evolution in the pufferfish have been debated in previous studies [13,27]. Aparicio et al. [13] suggested that the rapid deletion of nonfunctional sequences may be the predominant mechanism accounting for the compact genome of T. rubripes. Neafsey and Palumbi [27] proposed that a reduction in the rate of large insertions in the smooth puffer, rather than an increase in large deletions, can explain their genomic contraction and difference in size between the smooth and spiny puffer genomes. They also proposed that different transposable element activity might be the main driving force that is responsible for genome size variation between the smooth and spiny puffer genomes. In this study, although the difference amount of repeat elements itself was insufficient to explain the two-fold difference in genome size between $D$. holocanthus and smooth pufferfish, this might be due to that mutations have made many ancient transposable elements unrecognizable. However, we verified that amount and content of transposable ele- ments is different between $D$. holocanthus and the smooth pufferfish. Especially, LINEs with self transposable ability are more prevalent in $D$. holocanthus than that in smooth pufferfish, which means that transposable element activity is different between $D$. holocanthus and the smooth pufferfish. Therefore, our results indicated that the difference of transposable element activity contributed to and genome size variation between $D$. holocanthus and smooth pufferfish and that a reduction in the rate of large insertions caused by transposable elements is responsible to smooth pufferfish genomic contraction as proposed by Neafsey and Palumbi [27].

\section{Conclusions}

To study the genome size variation in the pufferfish, 10 BAC clones of the spiny pufferfish $D$. holocanthus were shotgun sequenced. In total, $776 \mathrm{~kb}$ of non-redundant sequences without gaps, constituting $0.1 \%$ of the D. holocanthus genome, were identified. Sequence analysis showed that $D$. holocanthus has a low gene density and repeat elements rich genome compared with the smooth pufferfish. Further analysis showed that D. holocanthus genome is characterized by longer introns and more interspersed repeats compared with the smooth pufferfish genomes. Our analysis also showed that the genome size variation between $D$. holocanthus and smooth pufferfish exhibits as the length variation in the homologous region and the different accumulation of the non-homologous sequence. Our results showed intron size variation was consistent with genome size variation between $D$. holocanthus and smooth pufferfish. We verified that different amount and content of repeat elements, especially the different accumulation of transposable elements, are responsible for the genome size variation between $D$. holocanthus and smooth pufferfish.

\section{Methods \\ BAC clone selection, shotgun sequencing, and assembly}

Ten BAC clones of around $100 \mathrm{~kb}$ were stochastically selected from a BAC library of the spiny pufferfish $D$. holocanthus and were sequenced using a standard shotgun strategy. Purified Escherichia coli genomic DNA-free plasmid DNA was sheared using a HydroShear (Genomic Solutions ${ }^{\circ}$ ). The resulting fragments were selected for a size range of 1.5-3.0 kb and extracted by agarose gel electrophoresis (QIAquick Gel Extraction Kit). The selected fragments were randomly inserted into the pUC118 vector (TaKaRa) with T4 ligase (Promega). Sequence reads of randomly selected sub-clones were generated from a single end using with the universal vector M13 primers.

After a sequence redundancy of more than threefold had been generated, a total of 9,286 high-quality reads were edited and assembled using the Phred/Phrap/ 
Consed program package [45-47]. The resultant contigs were then joined into scaffolds based on read-pair associations and order information from the shotgun clones.

Then interspersed repeats were then masked in the scaffolds of each BAC by RepeatMasker (version open3.1.5) using a repeat library (RepBase14.01) [48]. Masked scaffolds were compared with genomic sequences of $T$. rubripes and Te. nigroviridis using the Basic Local Alignment Search Tool (BLAST) algorithm with the default parameters[30]. The BLAST results were first clustered on the basis of colinearity for further screening. Manual inspection readily identified spurious hits which are mainly resulted from repetitive sequences and are not identified by RepeatMasker, and poor hits (identical base pairs < 100) arising from lineage divergence. After removing these spurious hits, we ordered and oriented as many super-scaffolds as possible within the same BAC clone by comparison with the genomes of $T$. rubripes and $T e$. nigroviridis, and the gaps were represented with 100 Ns.

\section{Sequence annotation and data analyses}

An integrative gene annotation process was performed mainly based on the Ensembl pipeline of gene annotation with some modifications [49]. Two ab initio gene prediction programs, FgenesH 2.6 [28] and GENSCAN 1.0 [29] were firstly adopted to make de novo gene prediction. TBLASTN [30] combined with GeneWise [31] analyses were then used for further gene predication. TBLASTN allows similarity searches against peptides database, and it was used with an E value of $1 \times 10^{-6}$ as the cutoff for identifying potential orthologous genes in the T. rubripes genome and the Te. nigroviridis genome in the Ensembl protein dataset (release 52). In total, 144 candidate peptides were identified in the T. rubripes genome and 67 in the Te. nigroviridis genome, and those showing more than $50 \%$ of identity and $70 \%$ of peptide length were maintained. This part of peptides was subsequently subjected to GeneWise analysis against the D. holocanthus genome sequence, taking into account splice sites and frameshifts. The best predictions were considered as overlapping results with above annotation steps and were integrated together to yield a summary of the annotated genes. This set of predictions was then manually filtered for redundancy resulted from alternative splicing or paralogous genes. The final predicted outputs were used as the input for the BLASTP [30] searches against the nonredundant GenBank protein database to assign their possible identity.

Genomic synteny were identified using BLASTN [30] searches between the sequenced $D$. holocanthus genome and the genomes of T. rubripes, Te. nigroviridis, G. aculeatus, and $O$. latipes. The criteria used to filter the BLASTN results were as follows: more than $50 \%$ identity and $70 \%$ of the query sequence length were maintained in the hit regions; more than two hits occurred between the query and the subject sequence, or if only one hit occurred, the hit length is longer than $300 \mathrm{bp}$. Homologous sequences were extracted using Perl scripts. The results were manually inspected and the synteny maps were visualized using the Artemis Comparison Tool (ACT) [50].

Repetitive element identification in D. holocanthus genome sequences was accomplished through RepeatMasker (version open-3.1.5), CENSOR [51], and BLAST similarity searches against known elements in REPBASE (version 14.01) [48] and GenBank. Repetitive elements were also identified using RepeatMasker (version open3.1.5) in the T. rubripes and Te. nigroviridis genome with their genome sequences from the Ensembl DNA dataset (release 52). The repeat elements in the homologous regions between the spiny pufferfish $D$. holocanthus and the smooth pufferfish were identified, according to the genomic synteny analyses.

\section{Additional material}

Additional file 1 Synteny maps. The figures show the synteny relationships between the BAC clone sequences of D. holocanthus and the smooth pufferfish T. rubripes and Te. nigroviridis genome.

\section{Authors' contributions}

SH and BG conceived the project. BG and XG performed the experiments. BG and $\mathrm{MZ}$ analyzed the data. BG and SH wrote the paper. All authors have read and approved the final manuscript.

\section{Acknowledgements}

We are very grateful to Jonathan F. Wendel, Corrinne Grover, Wenjing Tao, and three anonymous reviewers for their critical reading of and comments on this manuscript, which have allowed us to greatly improve the manuscript. This work was supported by the National Natural Science Foundation of China (Grant No. 30530120 and 2007CB411601) to Shunping He.

\section{Author Details}

${ }^{1}$ Fish Phylogenetics and Biogeography Group, Key Laboratory of Aquatic Biodiversity and Conservation, Institute of Hydrobiology, Chinese Academy of Sciences, Wuhan 430072, China, ${ }^{2}$ Graduate University of the Chinese Academy of Sciences, Beijing 100039, China and 'Wuhan Institute of Virology, Chinese Academy of Sciences, Wuhan 430071, China

Received: 28 March 2010 Accepted: 23 June 2010

Published: 23 June 2010

\section{References}

1. Thomas CA Jr: The genetic organization of chromosomes. Annual review of genetics Annu Rev Genet 1971, 5:237-256.

2. Claverie J-M: GENE NUMBER: What If There Are Only 30,000 Human Genes? Science (New York, NY) 2001, 291(5507):1255-1257.

3. Betran $E$, Long M: Expansion of genome coding regions by acquisition of new genes. Genetica 2002, 115(1):65-80.

4. Mirsky AE, Ris $\mathrm{H}$ : The desoxyribonucleic acid content of animal cells and its evolutionary significance. J Gen Physiol 1951, 34(4):451-462.

5. Gregory TR: Coincidence, coevolution, or causation? DNA content, cell size, and the C-value enigma. Biological reviews of the Cambridge Philosophical Society 2001, 76(1):65-101.

6. Boulesteix M, Weiss M, Biemont C: Differences in genome size between closely related species: the Drosophila melanogaster species subgroup. Mol Biol Evol 2006, 23(1):162-167. 
7. Jones RN, Brown LM: Chromosome evolution and DNA variation in Crepsis. Heredity 1976, 36:91-104.

8. Ohno S: Evolution by gene duplication. New York, Heidelberg. Berlin (Springer-Verlag); 1970.

9. Bennetzen JL: Mechanisms and rates of genome expansion and contraction in flowering plants. Genetica 2002, 115(1):29-36

10. Piegu B, Guyot R, Picault N, Roulin A, Saniyal A, Kim H, Collura K, Brar DS, Jackson S, Wing RA, Panaud O: Doubling genome size without polyploidization: dynamics of retrotransposition-driven genomic expansions in Oryza australiensis, a wild relative of rice. Genome Res 2006, 16(10):1262-1269

11. Deutsch M, Long M: Intron-exon structures of eukaryotic model organisms. Nucleic Acids Res 1999, 27(15):3219-3228.

12. Vinogradov AE: Intron-genome size relationship on a large evolutionary scale. J Mol Evol 1999, 49(3):376-384.

13. Aparicio S, Chapman J, Stupka E, Putnam N, Chia JM, Dehal P, Christoffels A, Rash S, Hoon S, Smit A, et al.: Whole-genome shotgun assembly and analysis of the genome of Fugu rubripes. Science (New York, NY) 2002, 297(5585):1301-1310

14. Zhou Q, Huang L, Zhang J, Zhao X, Zhang Q, Song F, Chi J, Yang F, Wang W: Comparative genomic analysis links karyotypic evolution with genomic evolution in the Indian muntjac (Muntiacus muntjak vaginalis). Chromosoma 2006, 115(6):427-436.

15. Zhang J: Evolution by gene duplication: an update. Trends Ecol Evol 2003, 18(6):292-298.

16. Hughes AL, Friedman R: Genome size reduction in the chicken has involved massive loss of ancestral protein-coding genes. Mol Biol Evol 2008, 25(12):2681-2688

17. Grover CE, Kim H, Wing RA, Paterson AH, Wendel JF: Microcolinearity and genome evolution in the AdhA region of diploid and polyploid cotton (Gossypium). Plant J 2007, 50(6):995-1006

18. Grover CE, Kim H, Wing RA, Paterson AH, Wendel JF: Incongruent patterns of local and global genome size evolution in cotton. Genome Res 2004, 14(8):1474-1482.

19. Grover CE, Hawkins JS, Wendel JF: Phylogenetic insights into the pace and pattern of plant genome size evolution. Genome dynamics 2008, 4:57-68.

20. Hawkins JS, Kim H, Nason JD, Wing RA, Wendel JF: Differential lineagespecific amplification of transposable elements is responsible for genome size variation in Gossypium. Genome Res 2006, 16(10):1252-1261

21. Wendel JF, Cronn RC, Alvarez I, Liu B, Small RL, Senchina DS: Intron size and genome size in plants. Mol Biol Evol 2002, 19(12):2346-2352.

22. Moriyama EN, Petrov DA, Hartl DL: Genome size and intron size in Drosophila. Mol Biol Evol 1998, 15(6):770-773.

23. Brainerd EL, Slutz SS, Hall EK, Phillis RW: Patterns of genome size evolution in tetraodontiform fishes. Evolution 2001, 55(11):2363-2368,

24. Jaillon O, Aury JM, Brunet F, Petit JL, Stange-Thomann N, Mauceli E, Bouneau L, Fischer C, Ozouf-Costaz C, Bernot A, et al:: Genome duplication in the teleost fish Tetraodon nigroviridis reveals the early vertebrate proto-karyotype. Nature 2004, 431(7011):946-957.

25. Hinegardner R, Rosen ED: Cellular DNA content and the evolution of teleostean fishes. The Am Nat 1972:621-644.

26. Holcroft NI: A molecular analysis of the interrelationships of tetraodontiform fishes (Acanthomorpha: Tetraodontiformes). Mol Phylogenet Evol 2005, 34(3):525-544.

27. Neafsey DE, Palumbi SR: Genome size evolution in pufferfish: a comparative analysis of diodontid and tetraodontid pufferfish genomes. Genome Res 2003, 13(5):821-830.

28. Solovyev VV, Salamov AA, Lawrence CB: Identification of human gene structure using linear discriminant functions and dynamic programming. Proc Int Conf Intell Syst Mol Biol 1995, 3:367-375.

29. Burge C, Karlin S: Prediction of complete gene structures in human genomic DNA. J Mol Bio/ 1997, 268(1):78-94.

30. Altschul SF, Madden TL, Schaffer AA, Zhang J, Zhang Z, Miller W, Lipman DJ: Gapped BLAST and PSI-BLAST: a new generation of protein database search programs. Nucleic Acids Res 1997, 25(17):3389-3402.

31. Birney E, Clamp M, Durbin R: GeneWise and Genomewise. Genome Res 2004, 14(5):988-995.

32. Grover CE, Yu Y, Wing RA, Paterson AH, Wendel JF: A phylogenetic analysis of indel dynamics in the cotton genus. Mol Biol Evol 2008 25(7):1415-1428.
33. Karlin S, Mrazek J: Compositional differences within and between eukaryotic genomes. P Natl Acad Sci USA 1997, 94(19):10227-10232.

34. Waterston RH, Lindblad-Toh K, Birney E, Rogers J, Abril JF, Agarwal P, Agarwala R, Ainscough R, Alexandersson M, An P, et al:. Initial sequencing and comparative analysis of the mouse genome. Nature 2002, 420(6915):520-562.

35. Chaudhury RC, Prasad R, Das CC: Chromosomes of six species of marine fishes. Caryologia 1979, 32(1):15-21

36. McLysaght A, Enright AJ, Skrabanek L, Wolfe KH: Estimation of synteny conservation and genome compaction between pufferfish (Fugu) and human. Yeast (Chichester, England) 2000, 17(1):22-36

37. Ogata H, Fujibuchi W, Kanehisa M: The size differences among mammalian introns are due to the accumulation of small deletions. FEBS letters 1996, 390(1):99-103.

38. Petrov DA, Sangster TA, Johnston JS, Hartl DL, Shaw KL: Evidence for DNA loss as a determinant of genome size. Science (New York, NY) 2000 287(5455):1060-1062.

39. SanMiguel P, Tikhonov A, Jin YK, Motchoulskaia N, Zakharov D, MelakeBerhan A, Springer PS, Edwards KJ, Lee M, Avramova Z, Bennetzen JL: Nested retrotransposons in the intergenic regions of the maize genome. Science (New York, NY) 1996, 274(5288):765-768.

40. Bennetzen JL: Transposable element contributions to plant gene and genome evolution. Plant Mol Biol 2000, 42(1):251-269.

41. Wong GK, Passey DA, Yu J: Most of the human genome is transcribed. Genome Res 2001, 11(12):1975-1977.

42. Deininger PL, Batzer MA: Mammalian retroelements. Genome Res 2002, 12(10):1455-1465

43. Deininger PL, Moran JV, Batzer MA, Kazazian HH Jr: Mobile elements and mammalian genome evolution. Curr Opin Genet Dev 2003, 13(6):651-658

44. Imai S, Sasaki T, Shimizu A, Asakawa S, Hori H, Shimizu N: The genome size evolution of medaka (Oryzias latipes) and fugu (Takifugu rubripes). Genes Genet Syst 2007, 82(2):135-144

45. Ewing B, Hillier L, Wendl MC, Green P: Base-calling of automated sequencer traces using phred. I. Accuracy assessment. Genome Res 1998, 8(3):175-185.

46. Ewing B, Green P: Base-calling of automated sequencer traces using phred. II. Error probabilities. Genome Res 1998, 8(3):186-194.

47. Gordon D, Abajian C, Green P: Consed: a graphical tool for sequence finishing. Genome Res 1998, 8(3):195-202.

48. Jurka J: Repbase update: a database and an electronic journal of repetitive elements. Trends Genet 2000, 16(9):418-420

49. Curwen V, Eyras E, Andrews TD, Clarke L, Mongin E, Searle SM, Clamp M: The Ensembl automatic gene annotation system. Genome Res 2004, 14(5):942-950

50. Carver TJ, Rutherford KM, Berriman M, Rajandream MA, Barrell BG, Parkhill J: ACT: the Artemis Comparison Tool. Bioinformatics 2005, 21(16):3422-3423

51. Jurka J, Klonowski P, Dagman V, Pelton P: CENSOR--a program for identification and elimination of repetitive elements from DNA sequences. Comput Chem 1996, 20(1):119-121.

52. Li C, Lu G, Ortí G: Optimal data partitioning and a test case for rayfinned fishes (Actinopterygii) based on ten nuclear loci. Syst Biol 2008 57(4):519-539.

53. Steinke D, Salzburger W, Meyer A: Novel relationships among ten fish model species revealed based on a phylogenomic analysis using ESTs. $J$ Mol Evol 2006, 62(6):772-784

54. Tyler JC, Santini F: Review and reconstructions of the tetraodontiform fishes from the Eocene of Monte Bolca, Italy, with comments on related Tertiary taxa. Studi e Ricerche sui Giacimenti Terziari di Bolca 2002, 9:47-119.

doi: 10.1186/1471-2164-11-396

Cite this article as: Guo et al., Genome size evolution in pufferfish: an insight from BAC clone-based Diodon holocanthus genome sequencing BMC Genomics 2010, 11:396 